\title{
Chromatin Immunoprecipitation and DNA Sequencing Identified a LIMS1/ILK Pathway Regulated by LMO1 in Neuroblastoma
}

\author{
NORIHISA SAEKI ${ }^{*}$, AKIRA SAITO $^{2}$, YUKI SUGAYA $^{2}$, MITSUHIRO AMEMIYA $^{2}$, \\ HIROE ONO $^{1}$, RIE KOMATSUZAKI ${ }^{3}$, KAZUYOSHI YANAGIHARA ${ }^{4}$ and HIROKI SASAKI ${ }^{3}$ \\ ${ }^{1}$ Division of Genetics, National Cancer Center Research Institute, Tokyo, Japan; \\ ${ }^{2}$ Statistical Genetics Analysis Division, StaGen Co. Ltd., Tokyo, Japan; \\ ${ }^{3}$ Department of Translational Oncology, National Cancer Center Research Institute, Tokyo, Japan; \\ ${ }^{4}$ Division of Pathology, Exploratory Oncology Research \& Clinical Trial Center, \\ National Cancer Center Hospital East, Chiba, Japan
}

\begin{abstract}
Background/Aim: Overall survival for the highrisk group of neuroblastoma (NB) remains at 40-50\%. An integrative genomics study revealed that LIM domain only 1 (LMO1) encoding a transcriptional regulator to be an NBsusceptibility gene with a tumor-promoting activity, that needs to be revealed. Materials and Methods: We conducted chromatin immunoprecipitation and DNA sequencing analyses and cell proliferation assays on two NB cell lines. Results: We identified three genes regulated by LMO1 in the cells, LIM and senescent cell antigen-like domains 1 (LIMS1), Ras suppressor protein 1 (RSU1) and relaxin 2 (RLN2). LIMS1 and RSU1 encode proteins functioning with integrin-linked kinase (ILK), and inhibition of LIMSI, ILK or RLN2 by shRNA reduced cell proliferation of the NB cells, which was also suppressed with an ILK inhibiting compound Cpd 22. Conclusion: The downstream of LMO1-regulatory cascade includes a tumor-promoting LIMSI/ILK pathway, which has a potential to be a novel therapeutic target.
\end{abstract}

NB is the most common extracranial solid tumor in childhood. Although accounting for more than $7 \%$ of

This article is freely accessible online.

*Present address: Division of Anatomy and Physiology, Okinawa Prefectural College of Nursing, Okinawa, Japan

Correspondence to: Norihisa Saeki, Division of Anatomy and Physiology, Okinawa Prefectural College of Nursing, Yogi 1-24-1, Naha, Okinawa 902-0076, Japan. Tel: +81 988338885, Fax: +81 988335133, e-mail: saeki@ okinawa-nurs.ac.jp

Key Words: Neuroblastoma, pediatric cancer, LMO1, ChIP-seq, therapeutic targets, transcription regulator. malignancies in patients younger than 15 , it is a cause for approximately $15 \%$ of all pediatric cancer deaths (1). NB cells develop from a sympathicoadrenal linage of the neural crest and form solid tumors in the adrenal medulla or paraspinal regions, which cause variable symptoms by compressing and/or invading to adjacent organs depending on their location (1-3). NB cells are heterogenous, and patient age at the time of diagnosis is one of the major factors that contribute to the determination of their biological characters; patients older than 18 months are considered to have a poorer prognosis when associated with dissemination than are patients younger than 18 months (1). In the context of prognosis, patients with NB are categorized into 4 groups: very low risk, low risk (LR), intermediate risk (IR) and high risk (HR), with a 5-year event-free survival of $>85 \%,>75$ to $\leq 85 \%, \geq 50$ to $\leq 75 \%$, and $<50 \%$, respectively, based on age at diagnosis, International Neuroblastoma Risk Group (INRG) tumour stage, histologic category, grade of tumor differentiation, DNA ploidy, and copy-number status at the MYCN oncogene locus and at chromosome 11q (1). Recent progress in NB treatment significantly improved patient prognosis; overall survival (OS) for LR and IR groups is now $>98 \%$ and 90 to $95 \%$, respectively. However, OS for the HR group still remains at 40 to $50 \%$, and 50 to $60 \%$ of these patients have a relapse, which, in that case, leaves no option of curable treatment (1-5). To improve the OS and quality of life for the HR group, the first and mandatory line of investigation is the identification of novel therapeutic target molecules in NB cells (6-8).

Currently, a number of genome-wide association studies (GWASs) have been performed for identifying disease susceptibility-associated genes using disease-related genetic variation, and the LIM domain only 1 ( $L M O 1)$ gene was identified as an NB susceptibility gene by a $\operatorname{GWAS}(9,10)$. 
The study also revealed that both the presence of an NBrelated allele of single nucleotide polymorphism (SNP) rs 110419 in the $L M O 1$ gene and the increased copy number of the gene contribute to augmented $L M O 1$ expression, and that LMO1 has a role in the proliferation of NB cells. Notably, the relation of the variation at rs110419 in LMOI as well as the increased copy number to NB is stronger in the HR group than in the non-HR. Thereafter, other studies also revealed the correlation between the SNP and NB susceptibility in different ethnic populations $(11,12)$. In addition, it was recently reported $L M O 1$ has synergetic effects with $M Y C N$ on NB metastasis (13). Therefore, it is highly likely that, as a transcription regulator, LMO1 is involved in the regulation of expression of variable genes important for NB progression in HR patients. Consequently, it is anticipated that identification of the LMO1's regulatory targets may uncover novel tumor-promoting molecular pathways in NB, in which novel therapeutic target molecules, especially for treatment of the HR group, will be discovered.

\section{Materials and Methods}

Cell lines. An NB cell line SK-N-SH (SH) was provided from European Collection of Cell Cultures and maintained in D-MEM (Wako Pure Chemical Industries, Ltd., Osaka, Japan) supplemented with $10 \%$ fetal calf serum (FCS). LA-N-5 (LAN5) was provided by the RIKEN Bioresource Center through the National Bio-Resource Project of the MEXT, Japan and maintained in RPMI1640 (Life Technologies, Tokyo, Japan) supplemented with $10 \%$ FCS.

\footnotetext{
Immune precipitation and western blot analyses. A $3 \times$ FLAG-LMO1 expression vector was constructed by insertion of LMO1 cDNA (14) into p3×FLAG-Myc-CMV (Sigma-Aldrich, St. Louis, MO USA). Cell lysates were prepared using CelLytic-M Mammalian Cell Lysis/Extraction Reagent (Sigma-Aldrich) and Protease Inhibitor Cocktail (Sigma-Aldrich). As preliminary experiment for selecting anti-LMO1 antibodies, immune precipitation was conducted on cell lysate of a gastric cancer cell line HSC-59 (14) introduced with a $3 \times$ FLAG-LMO1 expression construct, using anti-FLAG (EZView Red ANTI-FLAG M2 Affinity Gel, Sigma-Aldrich) and anti-LMO1 antibodies (Santa Cruz Biotechnology, Dallas, TX, USA). Immune blotting was performed with the anti-FLAG (Sigma-Aldrich) or anti-LMO1 (Santa Cruz Biotechnology) antibody as primary antibodies and HRP-conjugated anti-mouse or anti-goat antibodies (Santa Cruz Biotechnology) as secondary antibodies. The signals were detected by Pierce Western Blotting Substrate Plus (Thermo Fisher Scientific, Yokohama, Japan).
}

Real-time RT-PCR and microarray expression analyses. Total RNA was extracted from the NB cell lines with ISOGEN (WAKO). Quantitative RT-PCR was performed by converting about $5 \mu \mathrm{g}$ of total RNA to the first strand cDNA with High Capacity cDNA Reverse Transcription Kit (Life Technologies), followed by TaqMan Gene Expression Assay (Life Technologies, Applied Biosystems Assay ID: Hs00231133_m1 for LMO1, Hs00757864_m1 for LIMS1, Hs00765784_m1 for RSU1, Hs00754884_s1 for RLN2, and Pre-
Developed TaqMan Assay Reagent for glyceraldehyde-3-phosphate dehydrogenase $(G A P D H)$ ). The PCR was performed for 40 cycles under a condition of 2 steps of temperature: $95^{\circ} \mathrm{C}$ for $15 \mathrm{sec}$ and $60^{\circ} \mathrm{C}$ for $60 \mathrm{sec}$, using ABI PRISM 7900HT Sequence Detection System (Life Technologies). The relative transcript level was calculated using the $\mathrm{Ct}$ value of $G A P D H$ transcript as reference. The RNA was extracted from shRNA-introduced cells after 7-10 days' duration of G418 selection (Geneticin, WAKO). Microarray expression analyses were performed with GeneChip Human Genome U133 Plus 2.0 Array (Affymetrix, Santa Clara, CA, USA).

ChIP-seq. Chromatin samples of the NB cells were prepared using ChIP-IT Express Enzymatic Kit (Active Motif Japan, Tokyo, Japan), which were immunoprecipitated with the anti-LMO1 antibody and Protein G sepharose (GE Healthcare Japan). DNA samples purified from the precipitated chromatin were applied to the library construction using ChIP-Seq Sample Prep Kit (Illumina K.K., Tokyo, Japan) and Multiplexing Sample Preparation Oligonucleotide Kit (Illumina). In brief, after adapter ligation, around 300-bp fraction of DNA was isolated by electrophoresis through agarose gel, and clusters for sequencing were prepared using TruSeq PE Cluster Kit v3-cBot-HS (Illumina). DNA sequencing was conducted by Takara Bio Inc. (Shiga, Japan) with HiSeq 2000 (Illumina) for 100 nucleotides in a single-read manner. Input DNA samples were also sequenced as reference for data analyses.

Sequence data analyses. Quality check of the data was performed with FastQC (http://www.bioinformatics.babraham.ac.uk/projects/ fastqc/) which revealed a high quality of the sequence data (reads) and all reads were applied to alignment of the human genome. Raw sequencing reads were aligned to the UCSC hg19 reference genome using Burrows-Wheeler Aligner (15) supplemented with Novoalign (Novocraft Technologies, Selangor, Malaysia). As a result of the genome-alignment, 59,446,992 and 50,555,592 reads from the ChIP sequence data of SH and LAN5, respectively, were selected for peakidentification analyses, accompanied with their reference data. The peaks of accumulation of the reads were detected using six programs: CisGenome, MACS versions one and two, SISSRs, QuEST, and SPP. When using SISSRs, the $p$-value threshold was set to 0.1 and the threshold of the number of expected false-positive positions ( $e$-value) was set to 500. Peak detection using QuEST was conducted with three different parameter settings: parameters for transcription factor (QuEST-TF), for polII-like factor (QuEST-PolII), and for histone-type ChIP (QuEST-His). Peak detection using SPP was performed by two methods: window tag density (WTD) and mirror tag correlation (MTC). The $e$-value was set to 1000 when using SPP (WTD and MTC). The default settings were used for all other program options.

Down-regulation of LMO1 and target genes using shRNA. Each shRNA construct was prepared by annealing the (a) strand to the (b) as follows: for shLMO1 (target sequence: 5'-GGCATTGGACAAG TACTGG-3'), (a) 5'-tcgagGGtATTGGAtAAGTAtTGGttcaagagaCC AGTACTTGTCCAATGCC-tttttacgcgta-3' and (b) 5'-agcttacgc $\mathrm{g} t \mathrm{a}$ a a a G G C A T T G G A G A A G TA C T G G t c t c t t g a a CCAaTACTTaTCCAATaCCc-3', for shLIMS1 (target sequence: 5'GCATTATCCCAGAGAACGAA-3'), (a) 5'-tcgagGCATTgTC tCA GAGAgCGAAttcaagagaTTCGTTCTCTGGGATAATGCtttttacgcgt g-3' and (b) 5' -gatccacgcgtaaaaaaGCATTATCCCAGAGAAC GAAtctcttgaaTTCGcTCTCTGaGAcAATGCc-3', for shILK (target sequence: 5'-GGGACGCTGCTATGGACGAC-3'), (a) 5'-tcgag 


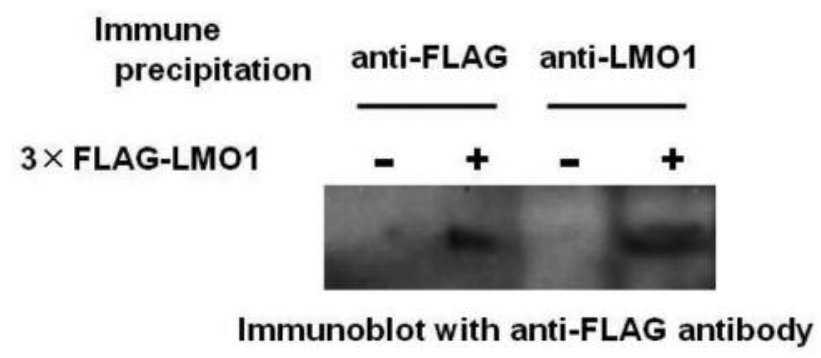

Figure 1. Immune precipitation revealed availability of an anti-LMOI antibody in immune precipitation. Immune precipitation was conducted on cell lysate of a gastric cancer cell line HSC-59 introduced with $3 \times F L A G-$ LMO1 expression construct, using an anti-FLAG and an anti-LMO1 antibodies. Immune blotting with an anti-FLAG antibody demonstrated $3 \times F L A G-L M O 1$ fusion protein in the precipitated specimens.

GGGAtGCTGCTgTGGAtGACttcaagagaGTCGTCCATAGCAGCG TCCCttttttacgcgtg-3' and (b) 5'-gatccacgcgtaaaaaaGGGACGCTG CTATGGACGACtctcttgaaGTCaTCCAcAGCAGCaTCCCc-3', for shRLN2 (target sequence: 5'-GCTAGGAGTCTGT TTACTAC-3'), (a) 5'-tcgagGtTAGGAGTCTGTTTgCTgCttcaagagaGTAGTAAA CAGACTCCTAGCttttttacgcgtg-3' and (b) 5'-gatccacgcgtaaaaaa GCTAGGAGTCTGTTTACTACtctcttgaaGcAGcAAACAGACTCC TAaCc-3', and for shGFP (target sequence: 5'-GCTACCT GTTCCATGGCCAA-3'), (a) 5'-tcgagGCTAtCTGTTCCgTGGCC gAttcaagagaTTGGCCATGGAACAGGTAGtttttacgcgtg -3 ' and (b) 5 ' - gatc cacgcg ta a a a aCTACCTGTTCCATGGCCAAtctct tgaaTcGGCCAcGGAACAGaTAGCc-3'. The constructs have a Mlu I site and cohesive ends for Xho I and BamH I or Hind III and possess alteration in the sense strand of the shRNA. Each shRNA was inserted into pLVSIN-CMV neo (Takara Bio) whose CMV promoter was replaced by hU6 promoter. The pLVSIN-hU6-shRNA constructs were introduced into Lenti- $\mathrm{X}^{\mathrm{TM}}$ 293T Cells (Takara Bio) using Lenti- $\mathrm{X}^{\mathrm{TM}}$ HTX Packaging System (Takara Bio) and after 72 hours' incubation, the medium was collected and its viral titer (infection units $/ \mathrm{ml}$ ) was determined by transduction to HT1080 cells. The NB cell lines were transducted with lentivirus $(>100,000$ infection units) in the presence of polybrene $(10 \mu \mathrm{g} / \mathrm{ml}$ in culture medium, Sigma-Aldrich).

Cell growth assay. For the analysis of the effect of the suppression of LMO1 or the product of the target genes, each cell line was seeded on a $100-\mathrm{mm}$ dish $\left(1 \times 10^{5}\right.$ cells/dish, in triplicate $)$ on the first day after transduced with the lentiviral particles containing the pLVSIN-hU6-shRNA construct, and was incubated for growth. The number of the cells on the dishes was counted on the fifth day of the assay. In the pathway inhibitory assay with Cpd22 (Merck Japan, Tokyo, Japan), the NB cells were prepared in five wells of 96-well plates (2000 cells /well). The inhibitors resolved in dimethylsulfoxide (DMSO, Merck Millipore) were added to the wells. The cells were incubated for 72 hours and the growth was evaluated by MTT (3-(4,5-dimethylthiazol-2-yl)-2,5-diphenyl tetrazolium bromide) assay using Cell Counting Kit-8 (Dojindo, Kumamoto, Japan).

Spheroid formation assay. On an Ultra Low Attachment 96-well plate (Corning Incorporated, NY, USA), NB cells $\left(1 \times 10^{3}\right.$ cells/well)
Table I. Summary of genome-mapping of ChIP-seq data.

\begin{tabular}{|c|c|c|c|c|}
\hline & \multicolumn{2}{|c|}{ SK-N-SH } & \multicolumn{2}{|c|}{ LA-N-5 } \\
\hline & $\begin{array}{l}\text { ChIP } \\
\text { DNA }\end{array}$ & $\begin{array}{c}\text { Reference } \\
\text { DNA }\end{array}$ & $\begin{array}{l}\text { ChIP } \\
\text { DNA }\end{array}$ & $\begin{array}{c}\text { Reference } \\
\text { DNA }\end{array}$ \\
\hline Sequenced reads & 60831674 & 52122693 & 51998018 & 55827557 \\
\hline BWA-mapped reads & 58867343 & 50890559 & 49645616 & 54622989 \\
\hline Novoalign-mapped reads & 579649 & 351511 & 909976 & 382795 \\
\hline Total mapped reads & 59446992 & 51242070 & 50555592 & 55005784 \\
\hline Unmapped & 1384682 & 880623 & 1442426 & 821773 \\
\hline Mappability (\%) & 97.72 & 98.31 & 97.23 & 98.53 \\
\hline
\end{tabular}

were incubated with $1 \mu \mathrm{M}$ Cpd22 in the medium, which was a $1: 1$ mixture of DMEM (Wako) and Ham's F12 (Wako) supplemented with epidermal growth factor (20 ng/ml, Sigma), basic fibroblast growth factor (20 ng/ml, Sigma) and B27 Supplement (Life Technology), or incubated with the solvent $0.01 \%$ DMSO, for five days. The number of spheroids per well was counted. The assay was conducted in quintuplicate.

\section{Results}

As the transcription regulator $L M O 1$ has no DNA-binding domain and therefore no consensus sequence for DNA binding, chromatin immunoprecipitation followed by DNA sequencing (ChIP-Seq) seemed to be superior to any other method for genome-wide identification of the LMO1's regulatory target genes. We performed ChIP-Seq on two NB cell lines expressing LMO1, SK-N-SH (SH), and LA-N-5 (LAN5), in which a cell-proliferation promoting activity of LMO1 was demonstrated in the previous study (9). The ChIP-Seq was performed with an anti-LMO1 antibody whose availability in immunoprecipitation had been confirmed (Figure 1). The immunoprecipitated DNA fragments and reference DNA fragments were sequenced by HiSeq2000. From the sequenced reads, those mapped to a reference genome sequence were selected for peak detection (Table I), and applied to 6 peak-detection programs with 9 different settings. Each program detected peaks (Table II), and, in this study, a definite peak was defined as the peaks detected by more than 5 of the 9 settings of the programs in each cell line. In the genome, many definite peaks were identified in which no gene was previously reported to reside. Although it is likely that these are true LMO1-binding sites and may have some biological significance, we focused on the definite peaks located in or near known genes (within a 5-kb proximity of known genes) encoding proteins. In the focused regions, we finally selected loci in which the definite peak was identified common to the two cell lines and obtained candidates for the target genes (Table III). To select the genes for further analyses, we examined the effect of 
SK-N-SH
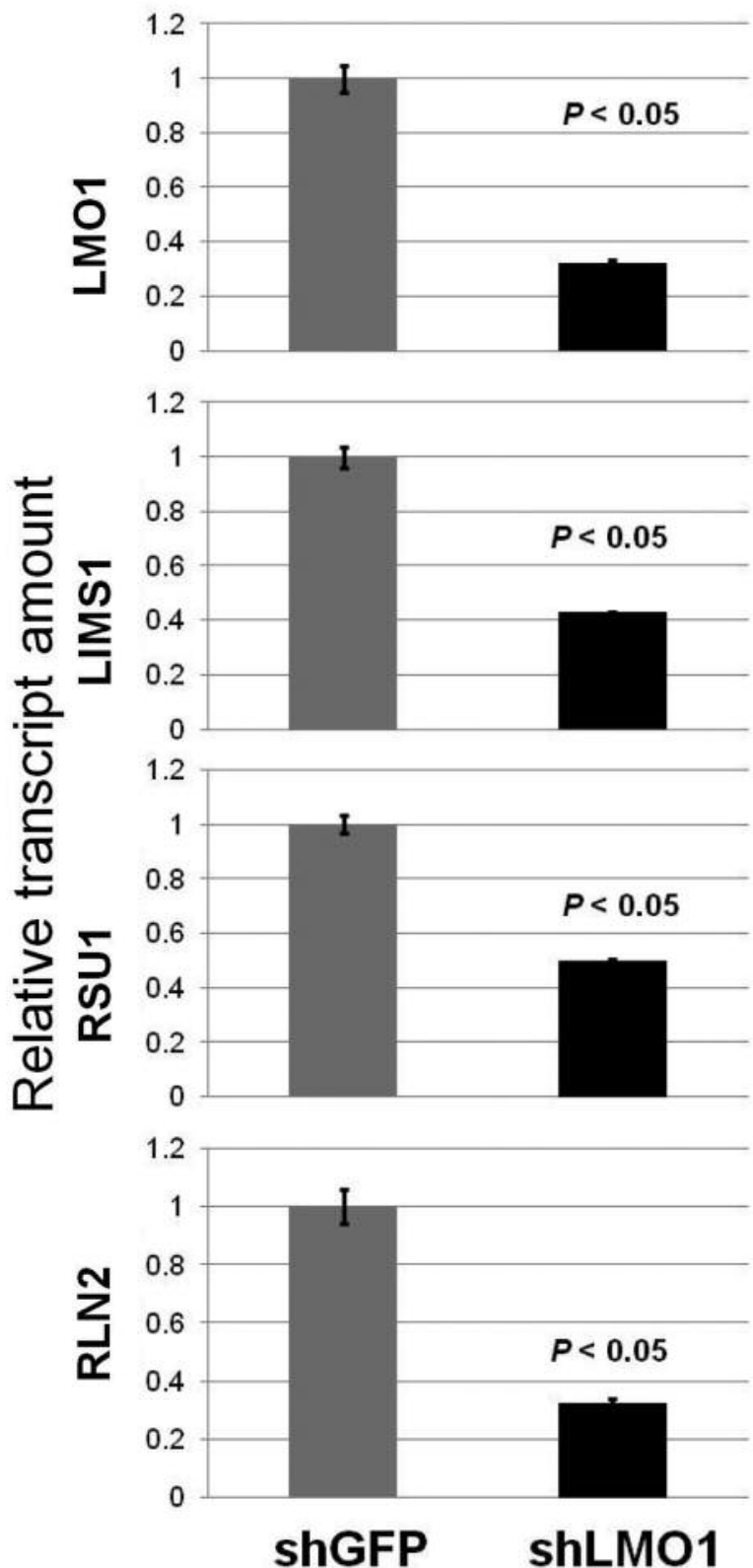

LA-N-5
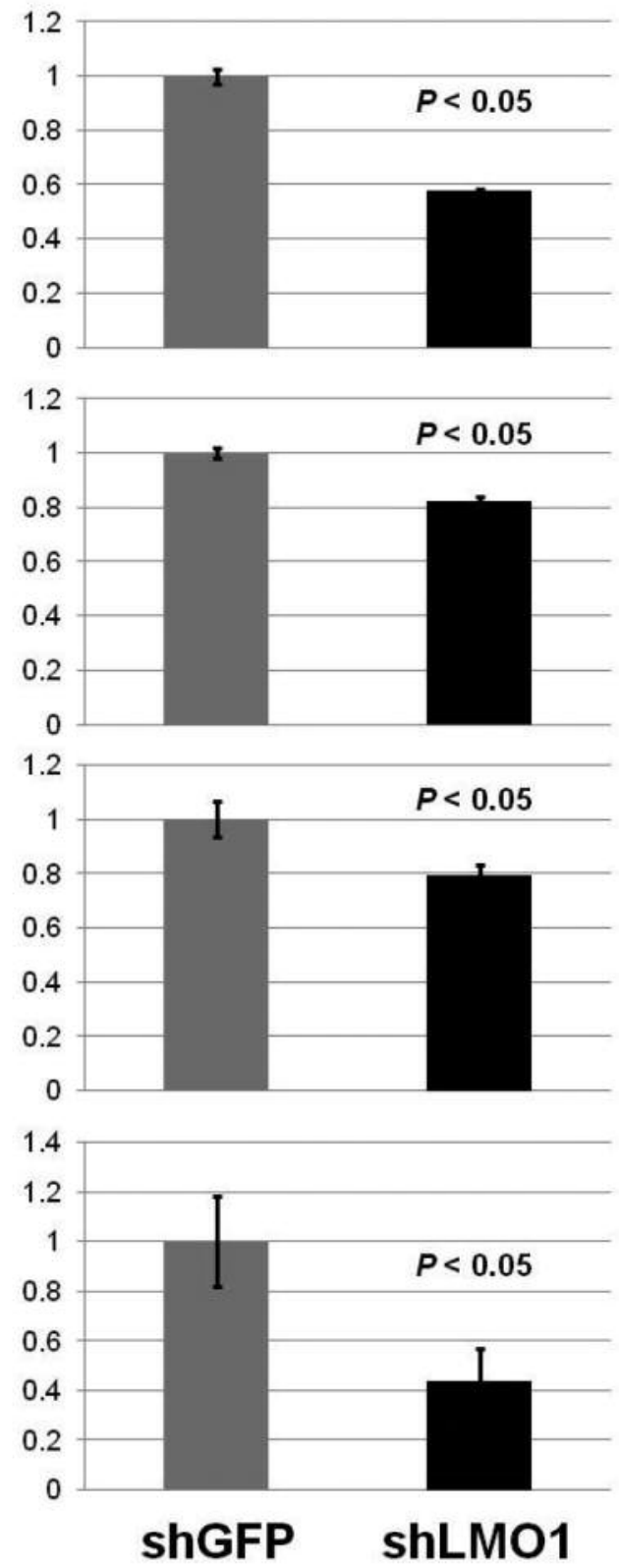

Figure 2. Down-regulation of LMO1 suppressed the expression of LIMS1, RSU1 and RLN2 genes. Down-regulation of LMO1 by shRNA resulted in suppression of LIMS1, RSU1 and RLN2 genes in SK-N-SH and LA-N-5 cells, when compared to cells introduced with shGFP (real-time PCR). Bar, Standard deviation, $P$, p-value of Student's t-test between the control and the subject.

Table II. Number of peaks detected by peak-detection programs.

\begin{tabular}{ccccccrrr} 
CisGenome & MACS & MACS & SISSRs & QuEST & QuEST & $\begin{array}{c}\text { QuEST } \\
\text {-His }\end{array}$ & $\begin{array}{c}\text { SPP/ } \\
\text {-PolII }\end{array}$ & $\begin{array}{c}\text { SPP/ } \\
\text { MTC }\end{array}$ \\
& & & & & & & \\
94714 & 28860 & 7023 & 85860 & 7438 & 3446 & 1859 & 45323 \\
40030 & 7930 & 1869 & 31714 & 2930 & 1399 & 51009 \\
& & & & & 7593 & 7938 \\
\hline
\end{tabular}




\section{SK-N-SH}

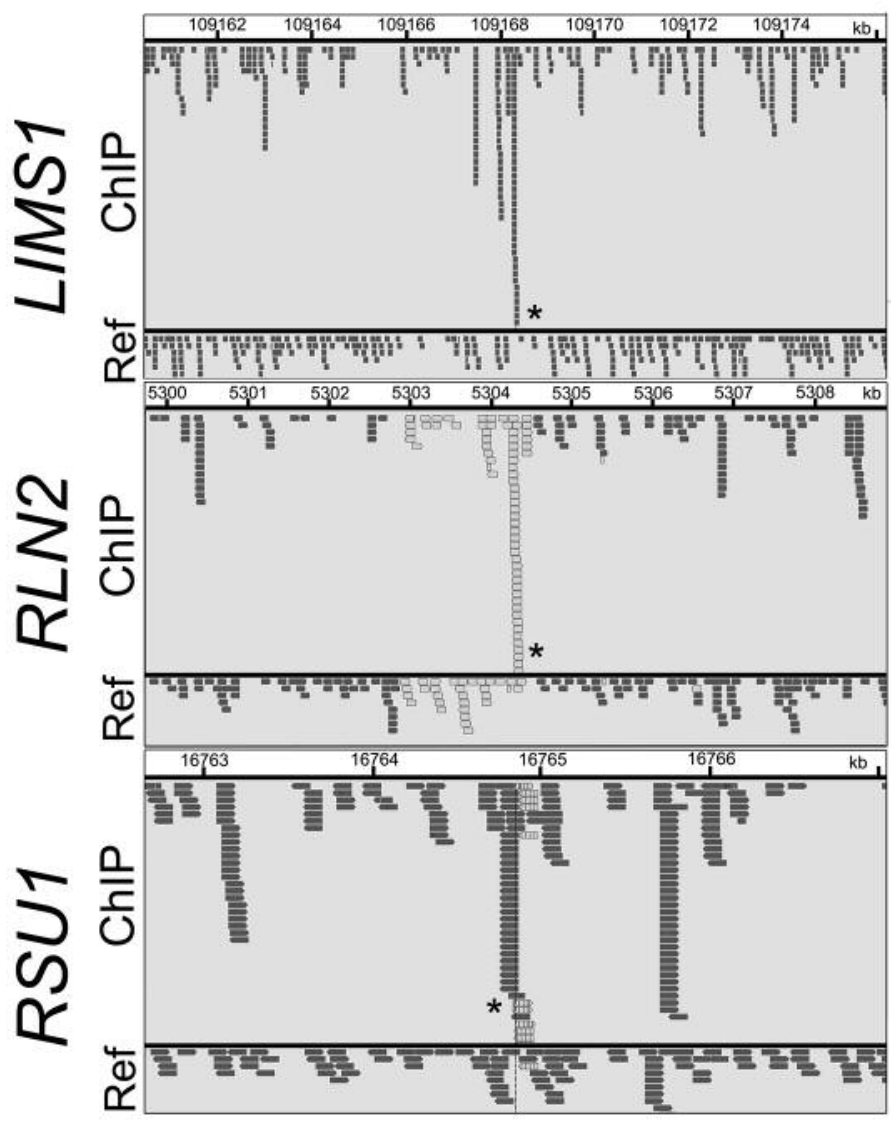

LA-N-5

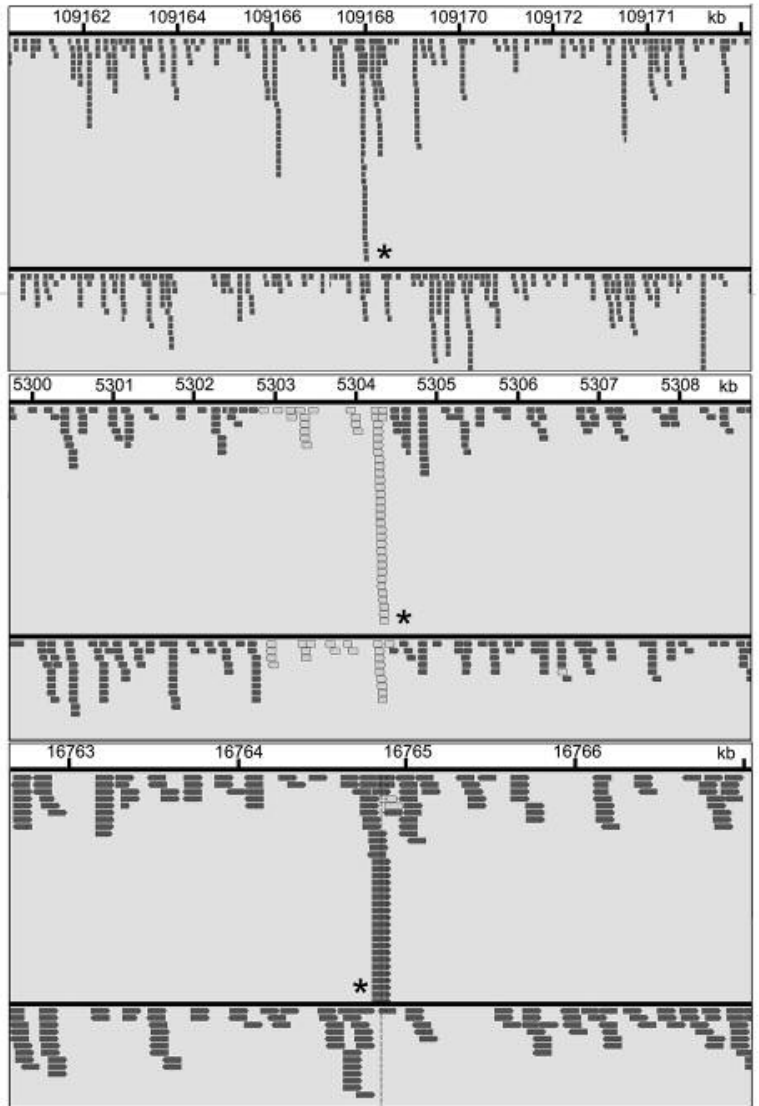

Figure 3. Peak-identification analyses on ChIP-seq data identified peaks in the regions harbouring LIMS1, RSU1 and RLN2. This figure shows the result of alignment analyses on ChIP-seq data and each small box corresponds to a read, which was drawn by using a software Integrative Genomics Viewer (30). The identified peaks are indicated by asterisks. ChIP, ChIP sample data, Ref, reference DNA data. Note that reads that are displayed with light gray borders and white fill, have a mapping quality equal to zero which means the read could also be mapped to another location.

LMO1 suppression on these genes by performing microarray expression analyses, comparing the original two NB cell lines and their counterparts in which LMO1 was suppressed with shRNA, which revealed that three genes, LIM and senescent cell antigen-like domains 1 (LIMS1), Ras suppressor protein $1(R S U 1)$ and relaxin 2 (RLN2), were down-regulated in the cell lines with LMO1 suppression (Table III). The down-regulation of the three genes was also observed in quantitative RT-PCR analyses (Figure 2), and accumulation of reads (peak) was demonstrated at the detected peak-identified regions for each gene in alignment analyses on the ChIP-seq data (Figure 3). Based on these results, we finally concluded that the three genes are direct regulatory targets of LMO1.

The ChIP-Seq identified three LMO1-target genes whose function in NB had not been thoroughly elucidated. Intriguingly, LIMS1 and RSU1 proteins have a role in integlin adhesome (16). LIMS1 protein, alternatively named $\mathrm{PINCH}$, has a role in several subcellular signallings at focal adhesions or integlin adhesomes, as a complex with integrinlinked kinase (ILK) and parvin (IPP complex). ILK is a $\beta 1$ integrin cytoplasmic domain interacting protein, and functions as a scaffold in forming multiprotein complexes connecting integrins to the actin cytoskeleton and signaling pathways, which is involved in the various oncogenic pathways related to cell proliferation, invasion, migration and angiogenesis (17). Some functions of RLN2 in carcinogenesis have been suggested, but remain to be investigated (18).

As the cell-proliferation promotion activity of LMO1 in the 2 NB cells was demonstrated in the previous study (9), we examined the consequences of suppression of the RLN2, LIMSI and ILK genes. This revealed that, when those genes were suppressed independently by shRNA, the 2 NB cells had reduced proliferation compared to the cells to which 
Table III. Candidates for LMO1-regulatd genes and their peak-detected regions identified by ChIP-seq analyses.

\begin{tabular}{|c|c|c|c|c|c|c|}
\hline \multirow{3}{*}{ Chromosome } & \multicolumn{3}{|c|}{ ChIP-Seq result } & \multicolumn{3}{|c|}{ Expression change by shLMO1 } \\
\hline & \multirow[t]{2}{*}{ Gene } & \multicolumn{2}{|c|}{ Peak-identified region* } & \multirow{2}{*}{$\begin{array}{l}\text { GeneChip } \\
\text { Probe }\end{array}$} & \multirow{2}{*}{$\begin{array}{c}\text { SK-N-SH } \\
\text { shLMO1 } \\
\text { /Control }\end{array}$} & \multirow{2}{*}{$\begin{array}{l}\text { LA-N-5 } \\
\text { shLMO1 } \\
\text { /Control }\end{array}$} \\
\hline & & Start & End & & & \\
\hline chr1 & $R F W D 2$ & 176101675 & 176102414 & 1552617_a_at & 1.07 & 1.33 \\
\hline \multirow{3}{*}{$\mathrm{chr} 2$} & SPAST & 32335801 & 32335811 & 207724 s at & 0.99 & 1.44 \\
\hline & & 32336003 & 32336111 & & & \\
\hline & & 32336238 & 32336264 & & & \\
\hline chr2 & LIMS1 & 109168312 & 109168349 & 207198_s_at & 0.68 & 0.81 \\
\hline chr2 & COBLL1 & 165674692 & 165674836 & 203641_s_at & $\mathrm{AC}$ & $\mathrm{AC}$ \\
\hline chr2 & COLAA3 & 228068131 & 228068457 & 214641_at & $\mathrm{AC}$ & $\mathrm{AC}$ \\
\hline \multirow[t]{2}{*}{ chr3 } & $S U M F 1$ & 4498194 & 4498251 & No probe & & \\
\hline & & 4498512 & 4498711 & & & \\
\hline chr3 & HACL1 & 15633532 & 15633651 & 223211_at & 1.07 & 1.07 \\
\hline chr3 & $S C N 5 A$ & 38691972 & 38692051 & 207413_s_at & $\mathrm{AC}$ & $\mathrm{AC}$ \\
\hline chr3 & $M O B P$ & 39510461 & 39510511 & 207659_s_at & $\mathrm{AC}$ & $\mathrm{AC}$ \\
\hline chr3 & $I L 17 R D$ & 57180001 & 57180142 & No probe & & \\
\hline $\operatorname{chr} 3$ & ЕРНА6 & 96548390 & 96548979 & No probe & & \\
\hline chr3 & KIAA1524 & 108294192 & 108294469 & 231855_at & 0.81 & 1.14 \\
\hline chr3 & FSTL1 & 120131193 & 120132019 & 208310_s_at & 1.15 & 1.18 \\
\hline \multirow[t]{2}{*}{ chr3 } & $K A L R N$ & 123828892 & 123829086 & 205635_at & 1.03 & 1.59 \\
\hline & & 123829197 & 123829399 & & & \\
\hline chr4 & C4orf 22 & 81311692 & 81311791 & No probe & & \\
\hline chr4 & COL25A1 & 109986212 & 109987206 & No probe & & \\
\hline \multirow[t]{2}{*}{ chr5 } & $R A S A I$ & 86582872 & 86583091 & 202677_at & 0.8 & 1.42 \\
\hline & & 86583641 & 86583778 & & & \\
\hline chr5 & FBXL17 & 107361298 & 107361536 & No probe & & \\
\hline chr5 & ADAMTS19 & 128885770 & 128886200 & 1553179_at & $\mathrm{AC}$ & $\mathrm{AC}$ \\
\hline \multirow[t]{3}{*}{ chr5 } & PCBD2 & 134260148 & 134260380 & 1554894_a_at & 1.11 & 1.4 \\
\hline & & 134262667 & 134262849 & & & \\
\hline & & 134263306 & 134263645 & & & \\
\hline chr6 & $C D Y L$ & 4895312 & 4895549 & 203099_s_at & $\mathrm{AC}$ & $\mathrm{AC}$ \\
\hline chr6 & LOC100506207 & 8531425 & 8531546 & No probe & & \\
\hline chr6 & $U B R 2$ & 42582052 & 42582091 & 212756_s_at & 0.89 & 1.26 \\
\hline chr7 & COL28A1 & 7406568 & 7406631 & No probe & & \\
\hline $\operatorname{chr} 7$ & SNX13 & 17844817 & 17844911 & 1553148_a_at & 1.21 & 0.74 \\
\hline chr7 & SEMA3E & 83018659 & 83019763 & 206941_x_at & $\mathrm{AC}$ & $\mathrm{AC}$ \\
\hline chr7 & C7orf60 & 112536022 & 112536590 & No probe & & \\
\hline \multirow[t]{2}{*}{ chr7 } & CNTNAP2 & 146171978 & 146172229 & 219302_s_at & $\mathrm{AC}$ & $\mathrm{AC}$ \\
\hline & & 147622288 & 147622573 & & & \\
\hline chr7 & RBM33 & 155466181 & 155466578 & No probe & & \\
\hline chr8 & LOC 100506990, LOC729732 & 12417252 & 12418491 & No probe & & \\
\hline chr8 & CSMD3 & 113932837 & 113933715 & No probe & & \\
\hline chr8 & TRPS1 & 116547401 & 116547544 & 218502_s_at & $\mathrm{AC}$ & $\mathrm{AC}$ \\
\hline chr8 & FER1L6 & 124867824 & 124868623 & No probe & & \\
\hline chr9 & RLN2 & 5304232 & 5304389 & 214519_s_at & 0.77 & 0.95 \\
\hline chr9 & MAPKAP1 & 128455743 & 128456019 & No probe & & \\
\hline $\operatorname{chr} 10$ & RSU1 & 16764832 & 16764871 & 201980_s_at & 0.95 & 0.97 \\
\hline $\operatorname{chr} 10$ & KIAA1217 & 24078991 & 24079136 & No probe & & \\
\hline chr11 & LUZP2 & 24947933 & 24948090 & No probe & & \\
\hline \multirow[t]{2}{*}{ chr11 } & TRAF6 & 36528751 & 36528949 & 205558_at & $\mathrm{AC}$ & $\mathrm{AC}$ \\
\hline & & 36529232 & 36529311 & & & \\
\hline chr11 & $D L G 2$ & 84902183 & 84902302 & 206253_at & $\mathrm{AC}$ & $\mathrm{AC}$ \\
\hline chr12 & FRS2 & 69951372 & 69951470 & 219907_at & 1.09 & $\mathrm{AC}$ \\
\hline chr13 & GPC5 & 93164452 & 93164511 & 207174_at & $\mathrm{AC}$ & $\mathrm{AC}$ \\
\hline chr13 & $N A L C N$ & 102049860 & 102049891 & 1554702_at & $\mathrm{AC}$ & $\mathrm{AC}$ \\
\hline chr14 & FUT8 & 66077790 & 66078160 & 203988_s_at & 1.06 & 0.96 \\
\hline chr16 & LOC283867 & 65499612 & 65499730 & No probe & & \\
\hline $\operatorname{chr} 17$ & RAD51C & 56803539 & 56804401 & 206066_s_at & 1.11 & 1.28 \\
\hline chr18 & SLC14A2 & 42816272 & 42816471 & 208409_at & $\mathrm{AC}$ & $\mathrm{AC}$ \\
\hline
\end{tabular}

*The position is based on UCSChg19. AC: Absent call. 

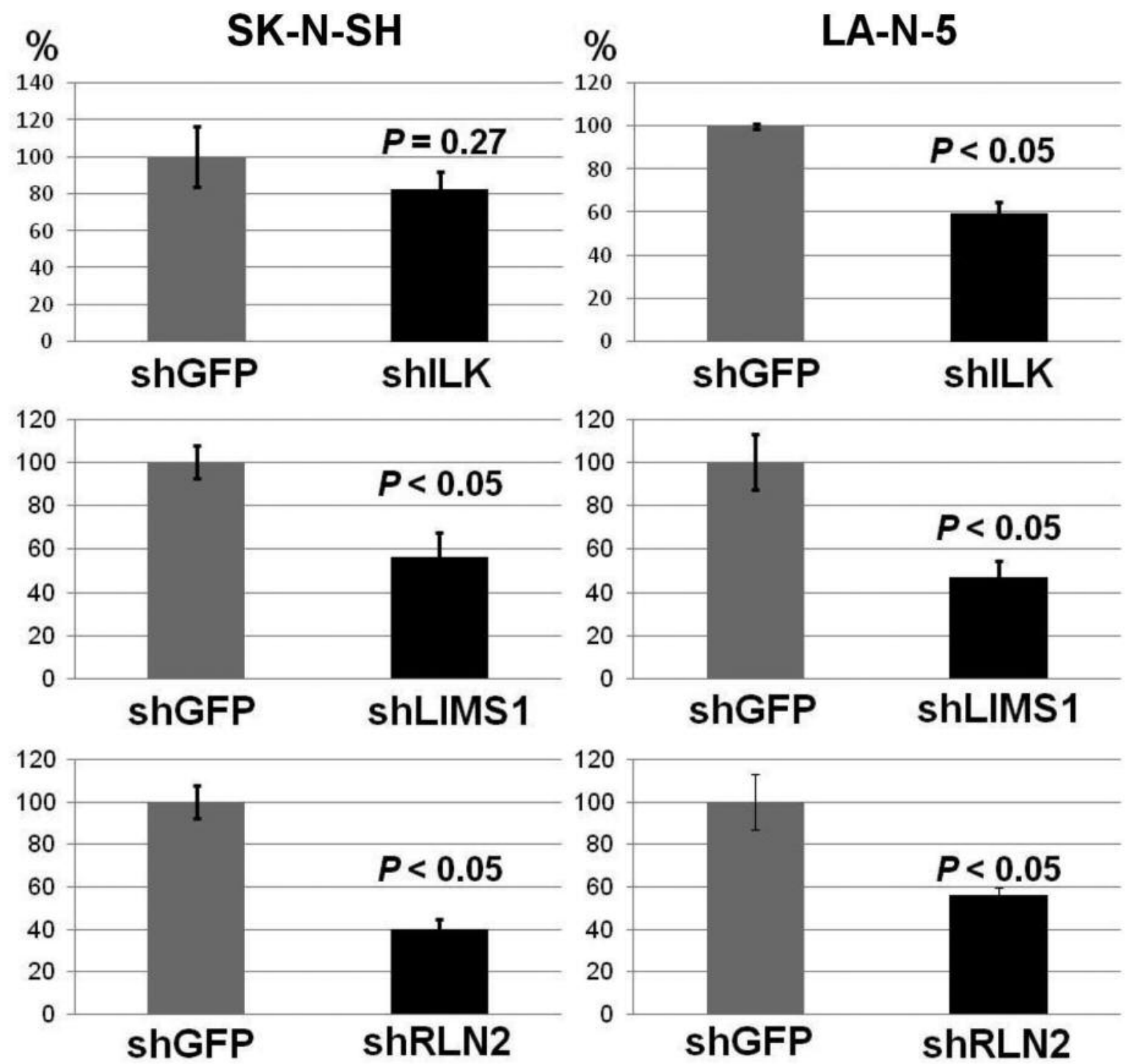

Figure 4. Down-regulation of ILK, LIMS1 or RLN2 genes suppressed proliferation of the NB cells. Down-regulation of each gene resulted in reduced proliferation of SK-N-SH and LA-N-5 cells, when compared to cells introduced with shGFP. Bar, Standard deviation, P, p-value of Student's t-test between the control and the subject.

shGFP was introduced, suggesting that each gene is involved in cell-proliferation promotion (Figure 4). Next, we tested the effect of Cpd22 (19), a commercially available inhibitor of the LIMS1/ILK pathway, on NB cell growth. The cell proliferation assays with 72-hour incubation demonstrated that $\mathrm{Cpd} 22$ reduced cell proliferation of the NB cell lines in vitro (Figure 5A). Moreover, it significantly suppressed anchorage-independent cell growth of the NB cells in a spheroid formation assay with a 5-day incubation (Figure $5 \mathrm{~B}$ ), suggesting treatment with $\mathrm{Cpd} 22$ could elicit a significant inhibitory effect on the NB cells.

\section{Discussion}

This study revealed that the LIMS1/ILK pathway is present in the downstream of the LMO1-regulatory cascade (Figure 6). LIMS1 protein has a role in several subcellular signallings at focal adhesions, as a complex with ILK and parvin (IPP complex), and the pathway involves several signallings related to carcinogenesis. Destabilization of the IPP complex enhances cell migration, which results from RAS signalling activation in tumorigenesis (20). Up-regulation of ILK is linked to EMT induction by thymosin $\beta 4$ in colorectal cancer cells (21). In 

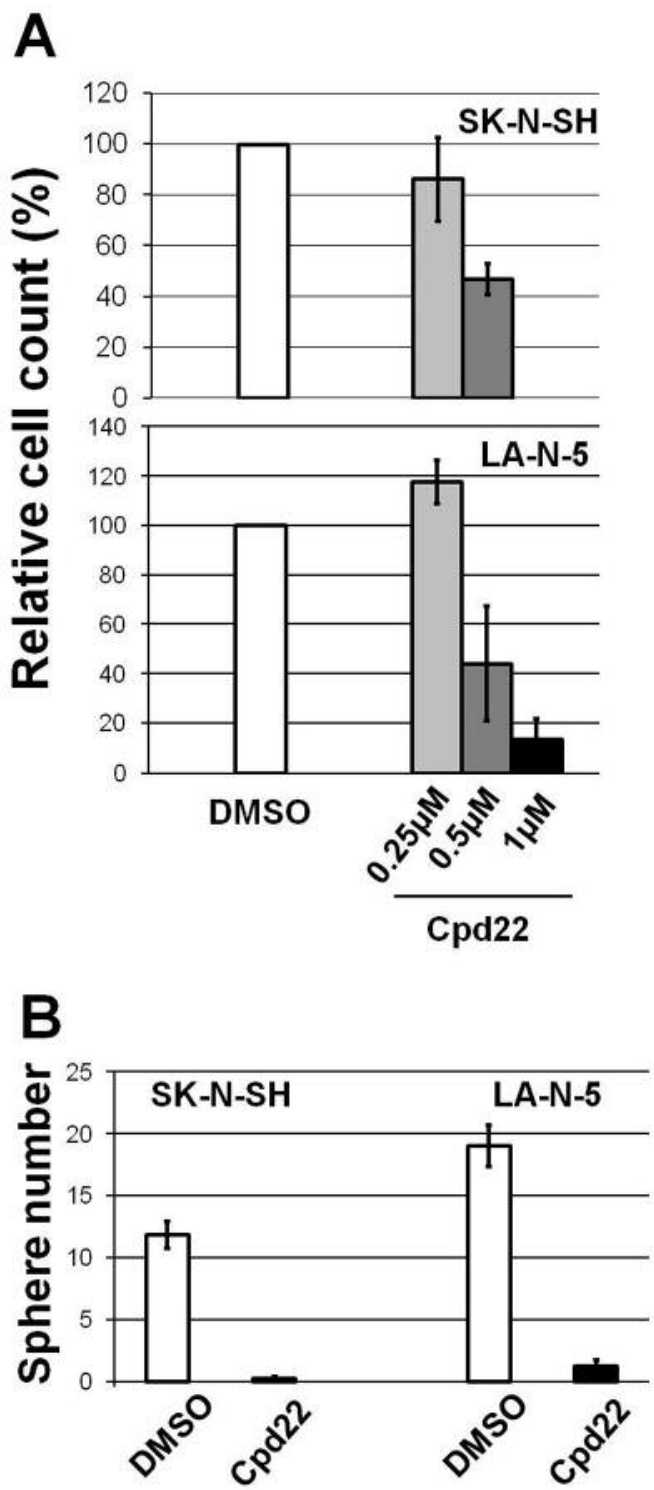

Figure 5. An ILK inhibitor Cpd22 reduced cell proliferation of the $N B$ cells. (A) Cpd22 reduced cell proliferation of SK-N-SH and LA-N-5 cells in dose dependent manner. The highest dose of the inhibitor showed statistically significant effect $(p<0.05)$ on the cell proliferation compared to DMSO. bar, standard deviation. (B) Cpd22 suppressed spheroid formation of the NB cells. bar, standard deviation.

addition, LIMS1 protects cancer cells from apoptosis via extracellular signal-regulated kinase 1/2 (ERK1/2) activation, and also contributes to radio- and chemo-resistance by promoting Akt1 (serine-threonine protein kinase) activation $(22,23)$. Moreover, the transforming growth factor $\beta$ (TGF $\beta$ ) up-regulates LIMS1, which induces epithelial-mesenchymal transition (EMT) in tubular cells of human kidney through down-regulation of E-cadherin and ZO-1 (Zona occludens 1)

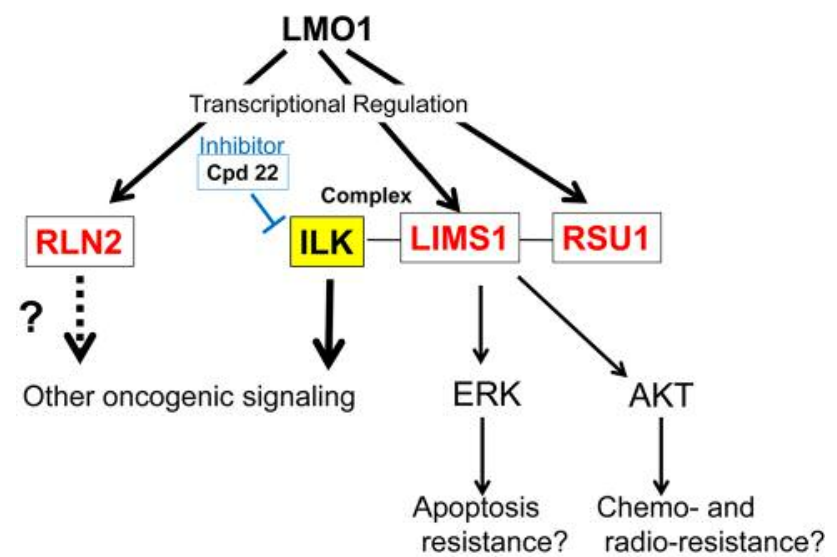

Figure 6. LMO1 regulates a LIMS1/ILK pathway. In this study, RLN2, RSU1 and LIMS1 genes are identified as LMO1's regulatory targets. RSU1 and LIMS1 proteins act as a complex with ILK protein in focal adhesion, which is known to transduce several oncogenic signals. A cell proliferation inhibition effect of an ILK inhibitor Cpd22 is demonstrated in this study.

depending on ILK activity (24). ILK was reported to be expressed in 33\% of Korean NB cases ( 3 in 9 cases) (25). In addition, supportive evidence was also obtained from clinical specimens. Microarray expression data set for 117 NB specimens (102 primary untreated neuroblastoma and 15 samples obtained at disease relapse) without $M Y C N$ amplification was available from ArrayExpress (E-GEOD3446, http://www.ebi.ac.uk/arrayexpress/experiments/EGEOD-3446/). The data set includes the expression data for three genes, LMOI, LIMSI and ILK, which shows that the three genes are expressed in all of the 117 NB specimens (data not shown). This suggests that these genes are expressed not only in the two cell lines, but also in a majority of clinical NB specimens. Although its precise function in NB remains to be elucidated, LMO1 was identified as an NB oncogene. Consequently, the LIMS1/ILK pathway is likely to have a critical role in NB carcinogenesis and a variety of potential therapeutic target molecules may be discovered in the pathway. Indeed, Cpd22 (1 $\mu \mathrm{M}$ in medium), an inhibitor of the LIMS1/ILK pathway showed cell-proliferation inhibition effects on the NB cell lines (Figure 5). The inhibitor previously showed the suppressive effect on prostate cancer cells: LNCaP $\left(\mathrm{IC}_{50}: 1 \mu \mathrm{M}\right)$ and PC-3 $(2 \mu \mathrm{M})$; and breast cancer cells: MDAMB-231 $(1 \mu \mathrm{M})$, MDA-MB-468 $(1.5 \mu \mathrm{M})$, SKBR3 $(1.8 \mu \mathrm{M})$ and MCF-7 $(2.5 \mu \mathrm{M})(19)$, while it significantly suppressed NB cell proliferation at $1 \mu \mathrm{M}$ in our study, suggesting NB will respond well to treatment with the inhibitor. As Cpd22 is orally bioavailable and its tumor growth suppression was demonstrated in a mouse prostate cancer-xenograft model (19), it can be promptly applied to NB xenograft mice. 
$R L N 2$ was reported to promote proliferation of cultured leiomyoma cells and prostate cancer cells $(26,27)$. It acted as an autocrine/paracrine factor and significantly increased anchorage-independent growth of thyroid carcinoma cell (28). In our study, shRLN2 decreased proliferation of the NB cells, which revealed, for the first time, the relevance of the gene to NB cell proliferation.

In this study, we searched for LMO1-target genes which are common to the $2 \mathrm{NB}$ cell lines, and identified 3 genes. However, each cell line could have distinct LMO1-targets, reflecting a clinical heterogeneity of NB. In addition, definitions of the detected peaks in this study, peaks detected in more than 5 of the 9 settings of the peak-identification programs, might be overly strict, which may have resulted in the exclusion of other true LMO1-binding genomic regions. Therefore, the extension of an analytical approach could reveal other novel therapeutic targets.

This study revealed the presence of the LIMS1/ILK pathway downstream of the LMO1-regulating cascade, and many previous studies reported a carcinogenesis-relevant function of this pathway. This study offered strong evidence supporting the hypothesis suggested by the previous GWAS that $L M O 1$ is an NB oncogene (9). As LMO1 is expressed in many NB cases, further studies on its downstream pathways should unveil other carcinogenesis-related pathways and molecules, which should lead to the development of a novel therapeutic strategy for HR-group NB patients. Intriguingly, other members of the LIMdomain-only proteins are known to be involved in oncogenic process of many types of cancer, and LMO3 is considered to be a neuroblastoma-associated oncogene, that could be a key molecule for discovery of novel oncogenic signalling in neuroblastoma (29).

\section{Acknowledgements}

This work was supported by the National Cancer Center Research and Development Fund (No. 23-B-18) and by JSPS KAKENHI Grant Number 26670510.

\section{References}

1 Maris JM, Hogarty MD, Bagatell $\mathrm{R}$ and Cohn SL: Neuroblastoma. Lancet 369: 2106-2120, 2007.

2 Maris JM: Recent advances in neuroblastoma. N Engl J Med 362: 2202-2211, 2010.

3 Cheung NK and Dyer MA: Neuroblastoma: developmental biology, cancer genomics and immunotherapy. Nat Rev Cancer 13: 397-411, 2013.

4 Morgenstern DA, Baruchel S and Irwin MS: Current and future strategies for relapsed neuroblastoma: challenges on the road to precision therapy. J Pediatr Hematol Oncol 35: 337-347, 2013.

5 Owens C and Irwin M: Neuroblastoma: The impact of biology and cooperation leading to personalized treatments. Crit Rev Clin Lab Sci 49: 85-115, 2012.
6 Song HY, Rellinger EJ, Park SH, Paul P, Qiao J, Vasilopoulos A, Ozden O, Gius D and Chung DH: Inhibition of sirtuin 6 induces neuroblastoma differentiation. Anticancer Res 38: 647-654, 2018.

7 Berger M and VON Schweinitz D: Therapeutic innovations for targeting childhood neuroblastoma: implications of the neurokinin1 receptor system. Anticancer Res 37: 5911-5918, 2017.

8 Abbou S, Lanvers-Kaminsky C, Daudigeos-Dubus E, LE Dret L, Laplace-Builhe C, Molenaar J, Vassal G, Geoerger B; within the ITCC Biology and Preclinical Evaluation Committee: Pololike kinase inhibitor volasertib exhibits antitumor activity and synergy with vincristine in pediatric malignancies. Anticancer Res 36: 599-609, 2016.

9 Wang K, Diskin SJ, Zhang H, Attiyeh EF, Winter C, Hou C, Schnepp RW, Diamond M, Bosse K, Mayes PA, Glessner J, Kim C, Frackelton E, Garris M, Wang Q, Glaberson W, Chiavacci R, Nguyen L, Jagannathan J, Saeki N, Sasaki H, Grant SF, Iolascon A, Mosse YP, Cole KA, Li H, Devoto M, McGrady PW, London WB, Capasso M, Rahman N, Hakonarson H and Maris JM: Integrative genomics identifies LMO1 as a neuroblastoma oncogene. Nature 469: 216-220, 2011.

10 Oldridge DA, Wood AC, Weichert-Leahey N, Crimmins I, Sussman R, Winter C, McDaniel LD, Diamond M, Hart LS, Zhu S, Durbin AD, Abraham BJ, Anders L, Tian L, Zhang S, Wei JS, Khan J, Bramlett K, Rahman N, Capasso M, Iolascon A, Gerhard DS, Guidry Auvil JM, Young RA, Hakonarson H, Diskin SJ, Look AT and Maris JM: Genetic predisposition to neuroblastoma mediated by a LMO1 super-enhancer polymorphism. Nature 528 : 418-421, 2015.

11 Zhang J, Lin H, Wang J, He J, Zhang D, Qin P, Yang L and Yan $\mathrm{L}$ : LMO1 polymorphisms reduce neuroblastoma risk in Chinese children: a two-center case-control study. Oncotarget 8: 6562065626, 2017.

12 He J, Zou Y, Wang T, Zhang R, Yang T, Zhu J, Wang F, Xia H: Genetic variations of GWAS-identified genes and neuroblastoma susceptibility: a replication study in southern Chinese children. Transl Oncol 10: 936-941, 2017.

13 Zhu S, Zhang X, Weichert-Leahey N, Dong Z, Zhang C, Lopez G, Tao T, He S, Wood AC, Oldridge D, Ung CY, van Ree JH, Khan A, Salazar BM, Lummertz da Rocha E, Zimmerman MW, Guo F, Cao H, Hou X, Weroha SJ, Perez-Atayde AR, Neuberg DS, Meves A, McNiven MA, van Deursen JM, Li H, Maris JM and Look AT: LMO1 Synergizes with MYCN to promote neuroblastoma initiation and metastasis. Cancer Cell 32: 310323, 2017.

14 Saeki N, Kim DH, Usui T, Aoyagi K, Tatsuta T, Aoki K, Yanagihara K, Tamura M, Mizushima H, Sakamoto H, Ogawa K, Ohki M, Shiroishi T, Yoshida T and Sasaki H: GASDERMIN, suppressed frequently in gastric cancer, is a target of LMO1 in TGF-betadependent apoptotic signalling. Oncogene 26: 6488-6498, 2007.

$15 \mathrm{Li} \mathrm{H}$ and Durbin R: Fast and accurate long-read alignment with Burrows-Wheeler transform. Bioinformatics 26: 589-595, 2010.

16 Winograd-Katz SE, Fässler R, Geiger B and Legate KR: The integrin adhesome: from genes and proteins to human disease. Nat Rev Mol Cell Biol 15: 273-288, 2014.

17 Hannigan G, Troussard AA and Dedhar S: Integrin-linked kinase: a cancer therapeutic target unique among its ILK. Nat Rev Cancer 5: 51-63, 2005.

18 Nair VB, Samuel CS, Separovic F, Hossain MA and Wade JD: Human relaxin-2: historical perspectives and role in cancer biology. Amino Acids 43: 1131-1140, 2012. 
19 Lee SL, Hsu EC, Chou CC, Chuang HC, Bai LY, Kulp SK and Chen CS: Identification and characterization of a novel integrinlinked kinase inhibitor. J Med Chem 54: 6364-6374, 2011.

20 Dougherty GW, Jose C, Gimona M and Cutler ML: The Rsu-1PINCH1-ILK complex is regulated by Ras activation in tumor cells. Eur J Cell Biol 87: 721-734, 2008.

21 Huang HC, Hu CH, Tang MC, Wang WS, Chen PM and Su Y: Thymosin beta4 triggers an epithelial-mesenchymal transition in colorectal carcinoma by upregulating integrin-linked kinase. Oncogene 26: 2781-2790, 2007.

22 Chen K, Tu Y, Zhang Y, Blair HC, Zhang L and Wu C: PINCH1 regulates the ERK-Bim pathway and contributes to apoptosis resistance in cancer cells. J Biol Chem 283: 2508-2517, 2008.

23 Eke I, Koch U, Hehlgans S, Sandfort V, Stanchi F, Zips D, Baumann M, Shevchenko A, Pilarsky C, Haase M, Baretton GB, Calleja V, Larijani B, Fässler R and Cordes N: PINCH1 regulates Akt1 activation and enhances radioresistance by inhibiting PP1alpha. J Clin Invest 120: 2516-2527, 2010.

$24 \mathrm{Li} \mathrm{Y,} \mathrm{Dai} \mathrm{C,} \mathrm{Wu} \mathrm{C} \mathrm{and} \mathrm{Liu} \mathrm{Y:} \mathrm{PINCH-1} \mathrm{promotes} \mathrm{tubular}$ epithelial-to-mesenchymal transition by interacting with integrinlinked kinase. J Am Soc Nephrol 18: 2534-2543, 2007.

25 Chung DH, Lee JI, Kook MC, Kim JR, Kim SH, Choi EY, Park SH and Song HG: ILK (beta1-integrin-linked protein kinase): a novel immunohistochemical marker for Ewing's sarcoma and primitive neuroectodermal tumour. Virchows Arch 433: 113-117, 1998.
26 Suzuki K, Nakabayashi K, Yamada AY, Lodhi RS, Hazama R, Ebina $\mathrm{Y}$ and Yamada $\mathrm{H}$ : Recombinant $\mathrm{H} 2$ relaxin inhibits apoptosis and induces cell proliferation in cultured leiomyoma cells without affecting those in cultured normal myometrial cells. Fertil Steril 97: 734-741, 2012.

27 Silvertown JD, Ng J, Sato T, Summerlee AJ and Medin JA: H2 relaxin overexpression increases in vivo prostate xenograft tumor growth and angiogenesis. Int J Cancer 118: 62-73, 2006.

28 Hombach-Klonisch S, Bialek J, Trojanowicz B, Weber E, Holzhausen HJ, Silvertown JD, Summerlee AJ, Dralle H, Hoang-Vu C and Klonisch T: Relaxin enhances the oncogenic potential of human thyroid carcinoma cells Am J Pathol 169: 617-632, 2006.

29 Matthews JM, Lester K, Joseph S and Curtis DJ: LIM-domainonly proteins in cancer. Nat Rev Cancer 13: 111-122, 2013.

30 Thorvaldsdóttir H, Robinson JT and Mesirov JP: Integrative Genomics Viewer (IGV): high-performance genomics data visualization and exploration. Brief Bioinform 14: 178-192, 2013.
Received January 6, 2018

Revised February 14, 2018

Accepted February 20, 2018 